\title{
Asymmetric modulation indices in full/partial response multi-H phase-coded modulations with different phase pulse functions
}

\author{
L.-S. Lee \\ H.-K. Hwang \\ S.-H. Chen
}

\begin{abstract}
A new concept of asymmetric modulation indices has recently been proposed and applied to full response multi-H phase-coded modulations (MHPM) with linear phase pulse function, and improved error probability performance has been found. In the paper, the above concept is extended in two directions. The partial response performance is analysed and compared with full response systems, and the effect of different phase pulse functions on bandwidth/power efficiency is discussed in detail. It is shown that not only is improved performance possible, but also a full range of design trade-offs, among bandwidth and power efficiencies and complexity, are available for system optimisation.
\end{abstract}

\section{Introduction}

Continuous phase modulation (CPM) $[1,2]$ signalling schemes have gained much interest in recent years due to their attractive spectral properties. The approach of phase-coded modulation provides good potential for further improvements in power and bandwidth efficiencies at the price of increased complexity [3]. Multi- $h$ phase-coded modulation (MHPM), described in detail by Anderson and Taylor [4], represents one trend in this area towards the development of efficient signalling schemes for the transmission of digital data as opposed to techniques such as minimum shift keying (MSK) or quaternary phase shift keying (QPSK) [5-9]. In the MHPM schemes, cyclically varying modulation indices are used in a prescribed manner so that the transmitted signal has phase slope variation, changing from one symbol interval to the next in response to the data symbols being transmitted. Since the phase function is altered in such a manner that unequal phase changes can result from transmission of the same data symbol in different contiguous intervals, the phase change that occurs in the first interval can not be undone during the second. The delays in the merging of neighbouring phase trellis

Paper 89891 (E7, E8), first received 11th June 1990 and in revised form 11th May 1992

L.-S. Lee is with the Department of Electrical Engineering, National Taiwan University, Taipei, Taiwan, Republic of China

H.-K. Hwang and S.-H. Chen are with the Institute of Communication Engineering, National Chiao Tung Universiy, Hsinchu, Taiwan, Republic of China paths will thus result in longer minimum Euclidean distances for MHPM schemes than those for MSK and hence provide the coding gain [10].

In practice, the modulation indices for MHPM are always restricted to be multiples of $1 / q$, where $q$ is an integer, and a finite number of phase states can therefore be used to demodulate the data in the receiver. In conventional MHPM schemes, the phase states at the transition times are all multiples of $\pi / q$, but not all possible phase states are used, i.e. only even or odd multiples of $\pi / q$ are used at any transition time $t=n T$. However, if all the phase states of multiples of $\pi / q$ could be used, we could have more flexibility in finding interesting signalling schemes, even if the constraint length in which the neighbouring phase trellis paths merge remains unchanged. In a recent paper [11] a new concept of using asymmetric modulation indices for bipolar data +1 and -1 , as compared to the symmetric indices used in conventional MHPM schemes, was proposed. In this new approach, the modulation indices $h_{+i}$ for the data +1 and $h_{-i}$ for the data -1 are not necessarily equal. More phase states and better flexibilities are therefore available for the designers to optimise the system performance. It was also shown [11] that essentially no bandwidth expansion and only a slight modification in implementation will be the result of this approach. However, the analysis and results [11] are only for full response systems with linear phase pulse functions. In this paper, the above concept of asymmetric indices for MHPM is extended in two directions. The partial response performance is analysed and compared with full response systems, and the effect of different phase pulse functions on bandwidth/power efficiency is discussed in detail.

\section{MHPM with asymmetric modulation indices}

The general form for an MHPM signal is

$$
s(t, \alpha)=\sqrt{ }(2 E / T) \cos \left[\omega_{c} t+\phi(t, \alpha)+\phi_{0}\right]
$$

where $E$ is the symbol energy, $T$ is the duration of a symbol, $\omega_{c}=2 \pi f_{c}$ is the carrier angular frequency and $\phi_{0}$ is an arbitrary carrier phase which, without loss of generality, can be set to be zero. The information-carrying phase function $\phi(t, \alpha)$ can be expressed as

$$
\phi(t, \alpha)=2 \pi \sum_{i=-\infty}^{\infty} a_{i} h_{i} q[t-(i-1) T] \quad-\infty \leqslant t \leqslant \infty
$$


where $x=\left\{\ldots, a_{-2}, a_{-1}, a_{0}, a_{1}, a_{2}, \ldots\right\}$ represents the sequence of data symbols, $h_{i}$ is the cyclically varying modulation index corresponding to the $i$ th symbol and $q(t)$ is a phase pulse function. In practice, the modulation indices are often obtained from a set of rational values of the form $\left\{l_{0} / q, l_{1} / q, \ldots, l_{K-1} / q\right\}$, where $l_{i}<q$ for $0 \leqslant i \leqslant K-1, l_{i}$ and $q$ are all integers. These indices are used cyclically with period $K$ so that

$$
h_{n K+j}=l_{j} / q \quad 0 \leqslant j \leqslant K-1 \text { and } n=0,1,2, \ldots
$$

The phase pulse function can be expressed as

$$
q(t)=\int_{-\infty}^{t} g(\tau) d \tau
$$

where $g(t)$ is a frequency pulse shape function with duration $L T$, i.e., it is zero for $t<0$ and $t>L T$, where $L$ is an integer, and nonzero otherwise. $L=1$ yields a full response signal, whereas $L>1$ corresponds to a partial response signal. In the previous paper [11], a full response linear phase pulse function is assumed, i.e.

$$
q(t)= \begin{cases}0 & t<0 \\ t / 2 T & 0 \leqslant t \leqslant T \\ 1 / 2 & t \geqslant T\end{cases}
$$

For conventional binary MHPM schemes, the modulation index $h_{i}$ has only one value for the $i$ th symbol no matter whether it is +1 or -1 , such that the phase trellis of the binary MHPM are always symmetric. Let $h_{+i}$ and $h_{-i}$ represent the indices $h_{i}$ for the $i$ th symbol being +1 and -1 , respectively. Apparently, $h_{-i}=h_{+i}$ for all $i$ in such a scheme. Assume two phase trajectories diverge from a given state at time $t=0$. The phase difference at time $t=T$ will be $\pi\left(h_{+1}+h_{-1}\right)=\pi\left(2 h_{1}\right)$ when $a_{1}$ 's are different, and $q\left(2 h_{i}\right)$ will be an even number. In the previous paper [11], a new concept of MHPM with asymmetric modulation indices corresponding to the bipolar data +1 and -1 was proposed, i.e. $h_{+i}$ and $h_{-i}$ are not necessarily equal.

This new asymmetric MHPM concept can provide the designer with an additional degree of freedom in choosing indices with better performance, because the separations among phase trajectories and the resulting minimum Euclidean distances depend on the values $h_{+i}$, $h_{-i}$ and $\left(h_{+i}+h_{-i}\right)$. For conventional MHPM, although $q\left(h_{+i}\right)$ and $q\left(h_{-i}\right)$ could be any chosen number, $q\left(h_{+i}\right.$ $+h_{-i}$ ) will always be an even number, and this constraint will limit the possibilities of maximising the minimum Euclidean distance. In other words, if $q\left(h_{+i}\right.$ $+h_{-i}$ ) does not have to be an even number, more opportunities will be available for designers to achieve better performance. This is the basic idea of the asymmetric MHPM concept. Extensive numerical results have been obtained for full response systems with linear phase pulse function in the previous paper [11], and it has been shown that with this approach significant performance improvements can be achieved compared to the conventional MHPM schemes.

\section{Performance analysis approaches}

In this paper, we will first analyse the bandwidth/power performance for full response MHPM with asymmetric indices with different phase pulse functions. The phase pulse functions assumed include linear phase (LP), raised-

IEE PROCEEDINGS-I, Vol. 139, No. 5, OCTOBER 1992 cosine (RC) and half-cycle sinusoid (HCS) functions:

$$
\begin{aligned}
& q(t)= \\
& \left\{\begin{array}{lll}
0 & t \leqslant 0 ; & \text { LP, RC, HCS } \\
t / 2 T & 0 \leqslant t \leqslant T ; & \text { LP } \\
{[1-\cos (\pi t / T)] / 4} & 0 \leqslant t \leqslant T ; & \text { HCS } \\
{[(t / T)-\sin (2 \pi t / T) / 2 \pi] / 2} & 0 \leqslant t \leqslant T ; & \text { RC } \\
1 / 2 & y \geqslant T ; & \text { LP, RC, HCS }
\end{array}\right.
\end{aligned}
$$

In the LP case, the frequency is held constant throughout the data interval, whereas with RC and HCS the instantaneous frequency varies slowly and smoothly. However, when the partial response schemes are analysed, only the linear phase pulse function will be assumed, i.e.

$$
q(t)= \begin{cases}0 & t<0 \\ t / 2 L T & 0 \leqslant t \leqslant L T \\ 1 / 2 & t \geqslant L T\end{cases}
$$

$L=1$ yields a full response signal as in eqn. 4 , whereas $L>1$ corresponds to a partial response signal. The performance for conventional partial response multi- $h$ schemes with such linear phase pulse function and $L=2$ have been found to be very attractive for $h_{i}<1.0$ [12]. Such a linear phase pulse function with duration $L T=2 T$ will thus be used in all the following numerical results for partial response systems.

It is difficult to analyse the error probability performance of a coherent receiver for MHPM schemes. One commonly used approach is that of calculating the minimum Euclidean distance over several symbol intervals for all possible cyclic shifts of $h_{i}$ values. In this paper, the minimum Euclidean distances will be used as an indicator of the power efficiency of MHPM schemes. The minimum Euclidean distance for minimum shift keying (MSK) is four.

When considering the spectral behaviour of MHPM schemes, the evaluation of the power spectra of MHPM signals is quite involved, thus simple approximations are often used to estimate the bandwidth of multi- $h$ signals, for example, the averaged modulation index $\bar{h}$ approach used by Wilson [13]. In this approach, the average of the modulation indices $h_{i}$ over one cycle is calculated

$$
\bar{h}=\frac{1}{2 K} \sum_{i=1}^{K}\left(h_{+i}+h_{-i}\right)
$$

Since the bandwidth of a constant- $h$ signal is proportional to its modulation index $h, \bar{h}$ can be taken as an estimate for the bandwidth of an asymmetric MHPM signal by considering a constant- $h$ signal with modulation index $\bar{h}$. In this paper, we also use $\bar{h}$ as an estimate for the bandwidth requirement of an MHPM signal with asymmetric indices, and $\bar{h}$ 's are rounded to 0.01 in the numerical results.

\section{Bandwidth/power performance analysis for full response cases with different phase pulse functions}

Here we first analyse the bandwidth/power performance for full response cases with different phase pulse functions. The minimum Euclidean distances for the best combinations of modulation indices with $K=2,3$, and 4 have been calculated. The maximum value of all the minimum Euclidean distances with the same $\bar{h}$ for all possible values of $q$ being considered is taken as a 
measure of the power efficiency and listed in Tables 1-3 with the value of $q$ for $K=2,3$ and 4, respectively. For $2-h$ schemes, it is clear that the largest minimum Euclidean distance is 7.81 with $\bar{h}=0.52$ and $q=13$ when the $\mathrm{RC}$ phase pulse function is used. Since the implementation complexity of a multi- $h$ system increases with $q, \mathrm{RC}$ phase $2-h$ schemes with $q=7$ and $\bar{h}=0.54$ is a good choice when the system complexity is considered.

Table 1: Maximum value of all the minimum Euclidean distances with the same averaged modulation index $\bar{h}$ for full response asymmetric MHPM with $\mathrm{K}=2$

\begin{tabular}{llll}
\hline $\bar{h}$ & \multicolumn{3}{l}{ Minimum Euclidean distance $(q)$} \\
\cline { 2 - 4 } & LP & RC & HCS \\
\hline 0.34 & $4.18(11)$ & $4.81(11)$ & $4.59(11)$ \\
0.35 & $4.51(12)$ & $5.13(12)$ & $4.92(12)$ \\
0.36 & $4.49(9)$ & $5.16(9)$ & $4.94(9)$ \\
0.37 & $4.79(13)$ & $5.40(13)$ & $5.19(13)$ \\
0.38 & $4.85(10)$ & $5.53(13)$ & $5.28(10)$ \\
0.39 & $5.14(11)$ & $5.78(11)$ & $5.56(11)$ \\
0.40 & $5.60(13)$ & $6.19(13)$ & $5.99(13)$ \\
0.41 & $5.37(8)$ & $6.05(8)$ & $5.82(8)$ \\
0.42 & $5.66(9)$ & $6.30(13)$ & $6.08(9)$ \\
0.43 & $6.09(11)$ & $6.65(11)$ & $6.46(11)$ \\
0.44 & $6.39(13)$ & $6.88(13)$ & $6.71(13)$ \\
0.45 & $6.10(11)$ & $6.84(11)$ & $6.59(11)$ \\
0.46 & $6.45(7)$ & $7.05(7)$ & $6.85(7)$ \\
0.47 & $6.87(10)$ & $7.31(10)$ & $7.16(10)$ \\
0.48 & $7.12(13)$ & $7.46(13)$ & $7.35(13)$ \\
0.50 & $3.98(13)$ & $3.96(13)$ & $3.97(13)$ \\
0.52 & $7.70(13)$ & $7.81(13)$ & $7.77(13)$ \\
0.53 & $7.57(9)$ & $7.73(9)$ & $7.68(9)$ \\
0.54 & $7.49(7)$ & $7.66(7)$ & $7.59(7)$ \\
0.55 & $7.31(11)$ & $7.58(11)$ & $7.49(11)$ \\
0.56 & $7.23(13)$ & $7.62(13)$ & $7.49(13)$ \\
0.57 & $7.14(11)$ & $7.59(11)$ & $7.44(11)$ \\
0.58 & $7.06(13)$ & $7.58(9)$ & $7.40(9)$ \\
0.59 & $6.99(8)$ & $7.58(8)$ & $7.38(8)$ \\
0.60 & $6.97(12)$ & $7.62(12)$ & $7.41(12)$ \\
0.61 & $6.96(11)$ & $7.49(11)$ & $7.42(11)$ \\
0.62 & $6.95(13)$ & $7.28(13)$ & $7.40(13)$ \\
0.63 & $7.00(13)$ & $7.33(10)$ & $7.49(13)$ \\
0.64 & $7.01(9)$ & $7.10(9)$ & $7.34(9)$ \\
0.65 & $7.04(12)$ & $7.10(12)$ & $7.34(12)$ \\
0.66 & $7.07(11)$ & $6.11(11)$ & $6.45(11)$ \\
0.67 & $7.15(12)$ & $6.44(12)$ & $6.75(12)$ \\
0.68 & $6.85(10)$ & $6.25(7)$ & $6.25(11)$ \\
0.69 & $7.34(12)$ & $6.44(12)$ & $6.75(12)$ \\
0.70 & $7.15(11)$ & $6.11(11)$ & $6.45(11)$ \\
0.71 & $7.11(13)$ & $6.05(13)$ & $6.39(13)$ \\
0.72 & $6.85(8)$ & $5.70(8)$ & $6.07(8)$ \\
0.73 & $6.85(10)$ & $5.70(10)$ & $6.07(10)$ \\
0.75 & $0.58(13)$ & $5.33(13)$ & $5.74(13)$ \\
\hline
\end{tabular}

From Tables 1 and 2 , it is interesting to find that the minimum Euclidean distance of the $2-h$ scheme with RC phase pulse function is larger than those of 3- $h$ schemes when $\bar{h}=0.52$. In other words, $2-h$ schemes can be used to obtain the error probability performance of $3-h$ schemes with less system complexity when $\bar{h}=0.52$. From these three tables, we find that the distances of 4- $h$ schemes are always larger than those of $2-h$ and 3- $h$ schemes, if the same values of $\bar{h}$ are used. Furthermore, it is found that the minimum Euclidean distance of the RC phase $4-h$ scheme with $\bar{h}=0.41$ is larger than all the distances in Tables 1 and 2, which means asymmetric 4- $h$ schemes can be used to obtain the error probability performance of 2- $h$ and 3- $h$ schemes with less bandwidth at the price of increased system complexity. Part of the optimum codes for 2- $h$ schemes are listed in Table 4. From this table, we find that most of the optimum codes with the same $\bar{h}$ for different phase functions are the same.
Table 2: The maximum value of all the minimum Euclidean distances with the same averaged modulation index $\bar{h}$ for full response asymmetric $M H P M$ with $K=3$

$\bar{h} \quad$ Minimum Euclidean distance $(q)$

LP RC HCS

$0.31 \quad 4.18(15) \quad 4.57$ (17) $4.40(17)$

$0.32 \quad 4.50(16) \quad 4.99(16) \quad 4.83(16)$

$0.33 \quad 4.27(15) \quad 4.76(15) \quad 4.60(15)$

$0.34 \quad 4.99(15) \quad 5.66(16) \quad 5.59(16)$

$0.35 \quad 5.28(17) \quad 5.89(17) \quad 5.71(17)$

$0.36 \quad 5.36(16) \quad 5.92(13) \quad 5.75(13)$

$0.37 \quad 5.64(17) \quad 6.27(15) \quad 6.15(15)$

$0.38 \quad 5.97(17) \quad 6.49(14) \quad 6.35(11)$

$0.39 \quad 6.08(14) \quad 6.94(14) \quad 6.77(14)$

$0.40 \quad 6.43(17) \quad 7.20(17) \quad 7.03(17)$

$\begin{array}{llll}0.41 \quad 6.56(17) & 7.20(17) \quad 7.03(17)\end{array}$

$0.426 .78(17) \quad 7.61(13) \quad 7.45(13)$

$0.43 \quad 7.24(17) \quad 7.75(16) \quad 7.59(16)$

$0.447 .16(15) \quad 7.55(17) \quad 7.41(17)$

$\begin{array}{lllll}0.45 & 7.54(17) & 7.89(17) & 7.86(17)\end{array}$

$\begin{array}{llll}0.46 & 7.79(16) \quad 7.89(17) & 7.86(17)\end{array}$

$\begin{array}{llll}0.47 & 7.72(11) & 7.97(17) & 7.87(17)\end{array}$

$0.48 \quad 7.80(11) \quad 8.04(14) \quad 8.01(11)$

$0.497 .90(17) \quad 8.03(14) \quad 8.13(17)$

$0.50 \quad 7.97(16) \quad 7.92(17) \quad 7.92(14)$

$0.517 .94(17) \quad 7.69(17) \quad 7.75(17)$

$0.527 .76(17) \quad 7.73(17) \quad 7.69(17)$

$0.537 .68(17) \quad 7.86(15) \quad 7.79(17)$

$0.547 .65(14) \quad 7.89(17) \quad 7.83(15)$

$\begin{array}{llll}0.55 & 7.70(16) \quad 7.89(17) \quad 7.86(17)\end{array}$

$0.56 \quad 7.80(17) \quad 7.98(16) \quad 7.89(16)$

$0.57 \quad 7.80(17) \quad 8.07(14) \quad 7.95(14)$

$\begin{array}{llll}0.58 & 7.80(17) & 8.19(12) & 8.05(12)\end{array}$

$0.59 \quad 7.97(16) \quad 8.48(16) \quad 8.31(16)$

$0.60 \quad 8.05(15) \quad 8.49(16) \quad 8.41(15)$

$0.618 .15(14) \quad 8.51(15) \quad 8.62(16)$

$\begin{array}{llll}0.62 & 8.43(17) & 8.52(14) & 8.77(15)\end{array}$

$0.638 .58(16) \quad 8.61(17) \quad 8.78(12)$

$\begin{array}{llll}0.64 & 8.82(14) \quad 8.45(16) & 8.80(17)\end{array}$

$\begin{array}{llll}0.65 & 8.97(16) & 8.49(16) & 8.81(14)\end{array}$

$\begin{array}{llll}0.66 & 8.97(16) & 8.30(17) & 8.79(17)\end{array}$

$0.67 \quad 5.86(15) \quad 5.59(15) \quad 5.67(15)$

$0.68 \quad 9.28(15) \quad 8.15(15) \quad 8.61(17)$

$0.69 \quad 9.28(15) \quad 8.05(17) \quad 8.55(16)$

$0.70 \quad 9.23(17) \quad 7.82(15) \quad 8.46(15)$

$0.71 \quad 9.23(17) \quad 7.77(17) \quad 8.42(17)$

$0.728 .85(16) \quad 7.62(16) \quad 8.07(16)$

$0.73 \quad 8.46(17) \quad 7.24(17) \quad 7.64(17)$

$0.74 \quad 8.46(17) \quad 7.24(17) \quad 7.64(17)$

$0.75 \quad 7.99(16) \quad 6.72(16) \quad 7.14(16)$

$0.76 \quad 7.68(17) \quad 6.32(17) \quad 7.76(17)$

$0.77 \quad 7.52(15) \quad 6.14(15) \quad 6.59(15)$

$0.78 \quad 7.19(16) \quad 5.76(16) \quad 6.22(16)$

$0.79 \quad 6.96(14) \quad 5.49(14) \quad 5.97(14)$

$0.806 .66(15) \quad 5.15(15) \quad 5.64(15)$

The best minimum Euclidean distances against the average of modulation indices $\bar{h}$ are plotted in Figs. $1-3$ for comparison. From Fig. 1, we find that for 2- $h$ schemes $(K=2)$ the minimum Euclidean distances of the RC phase pulse function are larger than those for LP and HCS functions when $\bar{h}$ is smaller than 0.6 . Note that the minimum Euclidean distances form a deep notch with the minimum value less than 4.0 when $\bar{h}=0.5$ for all the three phase functions considered. This is because for $2-h$ schemes the modulation index sets with $\bar{h}=0.5$ are weak index sets, i.e. the constraint length will be only 2 , instead of $K+1=3$ for these cases. We can also see that the highest power efficiency will be achieved at a minimum distance of 7.81 with a bandwidth parameter $\bar{h}=0.52$ when the RC phase pulse function is used, which seems to be a very attractive design. Apparently a plot such as in Fig. 1 provides very useful bandwidth-power performance analysis for design trade-offs and system optimisation.

IEE PROCEEDINGS-I, Vol. 139, No. 5, OCTOBER 1992 
Table 3: The maximum value of all the minimum Euclidean distances with the same averaged modulation index $\bar{h}$ for full response asymmetric MHPM with $K=4$

\begin{tabular}{|c|c|c|c|}
\hline \multirow[t]{2}{*}{$\bar{h}$} & \multicolumn{3}{|c|}{ Minimum Euclidean distance $(q)$} \\
\hline & LP & $\mathrm{RC}$ & $\mathrm{HCS}$ \\
\hline 0.31 & $5.32(22)$ & $5.77(22)$ & $5.59(19)$ \\
\hline 0.32 & $6.25(22)$ & $6.56(22)$ & $6.50(22)$ \\
\hline 0.33 & $6.27(20)$ & $6.45(20)$ & $6.39(20)$ \\
\hline 0.34 & $6.72(21)$ & $7.08(21)$ & $7.02(21)$ \\
\hline 0.35 & $6.89(21)$ & $7.21(22)$ & $7.04(22)$ \\
\hline 0.36 & $7.18(21)$ & $7.69(21)$ & $7.52(21)$ \\
\hline 0.37 & $7.18(21)$ & $7.69(21)$ & $7.52(21)$ \\
\hline 0.38 & $7.95(22)$ & $8.45(22)$ & $8.28(22)$ \\
\hline 0.39 & $8.36(22)$ & $8.77(22)$ & $8.67(22)$ \\
\hline 0.40 & $8.54(22)$ & $8.77(22)$ & $8.69(22)$ \\
\hline 0.41 & $9.23(19)$ & $9.59(22)$ & 9.50 (19) \\
\hline 0.42 & $9.16(21)$ & $9.22(21)$ & $9.32(21)$ \\
\hline 0.43 & $8.90(21)$ & $8.79(22)$ & $8.72(21)$ \\
\hline 0.44 & $8.51(21)$ & $8.58(22)$ & $8.50(20)$ \\
\hline 0.45 & $8.76(21)$ & $8.45(21)$ & $8.56(21)$ \\
\hline 0.46 & $8.46(20)$ & $8.18(20)$ & $8.27(20)$ \\
\hline 0.47 & $8.19(22)$ & $8.33(22)$ & $8.24(22)$ \\
\hline 0.48 & $8.54(22)$ & $8.84(20)$ & $8.73(20)$ \\
\hline 0.49 & $8.90(21)$ & $9.33(21)$ & $9.18(21)$ \\
\hline 0.50 & $7.84(21)$ & $7.88(22)$ & $7.90(22)$ \\
\hline 0.51 & $9.23(19)$ & $9.63(22)$ & 9.50 (19) \\
\hline 0.52 & $9.62(22)$ & $9.83(20)$ & $9.87(20)$ \\
\hline 0.53 & $10.01(22)$ & $9.59(22)$ & $9.75(22)$ \\
\hline 0.54 & $9.68(21)$ & $9.17(21)$ & $9.37(21)$ \\
\hline 0.55 & $9.62(22)$ & 8.92 (19) & $9.11(22)$ \\
\hline 0.56 & $9.24(18)$ & $8.63(22)$ & $8.87(18)$ \\
\hline 0.57 & $8.84(21)$ & $8.49(22)$ & $8.46(21)$ \\
\hline 0.58 & 8.55 (17) & $8.62(20)$ & $8.66(22)$ \\
\hline 0.59 & $8.74(20)$ & $9.02(20)$ & $8.93(20)$ \\
\hline 0.60 & 9.04 (18) & $9.33(21)$ & $9.18(21)$ \\
\hline 0.61 & $9.50(21)$ & $9.63(22)$ & $9.50(21)$ \\
\hline 0.62 & $9.79(22)$ & $9.90(18)$ & $9.96(22)$ \\
\hline 0.63 & $9.95(20)$ & $10.02(21)$ & $10.19(21)$ \\
\hline 0.64 & $10.28(22)$ & $9.48(17)$ & $9.86(21)$ \\
\hline 0.65 & $10.12(18)$ & $9.70(22)$ & $9.96(22)$ \\
\hline 0.66 & $10.39(21)$ & $9.54(20)$ & $9.95(20)$ \\
\hline 0.67 & $10.39(21)$ & $9.17(21)$ & $9.57(21)$ \\
\hline 0.68 & $10.07(21)$ & $9.02(19)$ & $9.11(22)$ \\
\hline 0.69 & $9.96(22)$ & $8.70(22)$ & $9.11(22)$ \\
\hline 0.70 & $9.96(22)$ & $8.61(22)$ & $9.11(22)$ \\
\hline 0.71 & $9.96(22)$ & $8.31(20)$ & $8.91(20)$ \\
\hline 0.72 & $9.79(19)$ & $8.12(21)$ & 8.64 (18) \\
\hline 0.73 & $9.77(20)$ & 7.80 (19) & $8.44(21)$ \\
\hline 0.74 & $9.46(21)$ & 7.62 (17) & 8.21 (17) \\
\hline 0.75 & $7.98(22)$ & $7.44(20)$ & $7.90(22)$ \\
\hline 0.76 & $9.08(22)$ & $7.30(22)$ & $8.08(22)$ \\
\hline 0.77 & $8.54(21)$ & 7.11 (21) & $7.58(21)$ \\
\hline 0.78 & $8.30(19)$ & 6.86 (19) & 7.33 (19) \\
\hline 0.79 & $7.96(20)$ & $6.48(20)$ & $6.96(20)$ \\
\hline 0.80 & $7.66(21)$ & $6.15(21)$ & $6.64(21)$ \\
\hline
\end{tabular}

In Fig. 2, it can be seen that for 3- $h$ schemes $(K=3)$, although the RC phase pulse function seems to be the most power efficient when $\bar{h}$ is less than 0.6 , the LP phase pulse function will be the best choice when $\bar{h}$ is allowed to exceed 0.65 . In other words, LP can be used to achieve better error probability performance but at the price of a slight bandwidth expansion. It can also be seen that for 3- $h$ schemes the modulation index sets with $\bar{h}=0.67$ will limit the constraint length to be 3 instead of $K+1=4$ and this is why the minimum Euclidean distances form another deep notch there. On the other hand, LP phase pulse function with $\bar{h}=0.68$ can be used to achieve the largest distance of 9.28 for $3-h$ schemes. It is clear that the choice of $\bar{h}$ value is very important for the system design of MHPM schemes.

For $4-h$ schemes $(K=4)$ in Fig. 3, the weak modulation index sets have $\bar{h}=0.5$, although the notch is not so deep as before. From this Figure, we find that the minimum Euclidean distances for $\mathrm{RC}$ phase pulse func-
Table 4: The maximum value of all the minimum Euclidean distances with the same averaged modulation index $\bar{h}$ for 2-h schemes with $q$

$\bar{h} \quad$ Minimum Euclidean distance $(q)$ $\left(A_{1}, A_{2}\right)$

\begin{tabular}{|c|c|c|c|c|c|c|}
\hline & \multicolumn{2}{|c|}{ LP } & \multicolumn{2}{|c|}{$\mathrm{RC}$} & \multicolumn{2}{|c|}{ HCS } \\
\hline & conv. & asym. & conv. & asym. & conv. & asym. \\
\hline 0.54 & $\begin{array}{l}7.36(12) \\
(12,14)\end{array}$ & $\begin{array}{l}7.49(7) \\
(7,8)\end{array}$ & $\begin{array}{l}7.61(12) \\
(12,14)\end{array}$ & $\begin{array}{l}7.66(7) \\
(7,8)\end{array}$ & $\begin{array}{l}7.53(12) \\
(12,14)\end{array}$ & $\begin{array}{l}7.59(7) \\
(7.8)\end{array}$ \\
\hline 0.55 & $\begin{array}{l}7.25(10) \\
(10,12)\end{array}$ & $\begin{array}{l}7.31(11) \\
(11,13)\end{array}$ & $\begin{array}{l}7.54(10) \\
(10,12)\end{array}$ & $\begin{array}{l}7.58(11) \\
(11,13)\end{array}$ & $\begin{array}{l}7.45(10) \\
(10,12)\end{array}$ & $\begin{array}{l}7.49(11) \\
(11,13)\end{array}$ \\
\hline 0.56 & $\begin{array}{l}7.10(8) \\
(8,10)\end{array}$ & $\begin{array}{l}7.23(13) \\
(14,15)\end{array}$ & $\begin{array}{l}7.45(8) \\
(8,10)\end{array}$ & $\begin{array}{l}7.62(13) \\
(14,15)\end{array}$ & $\begin{array}{l}7.33(8) \\
(8,10)\end{array}$ & $\begin{array}{l}7.49(13) \\
(14,15)\end{array}$ \\
\hline 0.57 & $\begin{array}{l}6.65(7) \\
(6,10)\end{array}$ & $\begin{array}{l}7.14(11) \\
(12,13)\end{array}$ & $\begin{array}{l}6.34(7) \\
(6,10)\end{array}$ & $\begin{array}{l}7.59(11) \\
(12,13)\end{array}$ & $\begin{array}{l}6.66(7) \\
(6,10)\end{array}$ & $\begin{array}{l}7.44(11) \\
(12,13)\end{array}$ \\
\hline 0.58 & $\begin{array}{l}7.06(13) \\
(14,16)\end{array}$ & $\begin{array}{l}7.06(13) \\
(14,16)\end{array}$ & $\begin{array}{l}7.55(13) \\
(14,16)\end{array}$ & $\begin{array}{l}7.58(9) \\
(10,11)\end{array}$ & $\begin{array}{l}7.38(13) \\
(14,16)\end{array}$ & $\begin{array}{l}7.40(9) \\
(10.11)\end{array}$ \\
\hline 0.59 & $\begin{array}{l}6.98(11) \\
(12,14)\end{array}$ & $\begin{array}{l}6.99(8) \\
(9,10)\end{array}$ & $\begin{array}{l}7.49(11) \\
(12,14)\end{array}$ & $\begin{array}{l}7.58(8) \\
(9,10)\end{array}$ & $\begin{array}{l}7.35(11) \\
(12,14)\end{array}$ & $\begin{array}{l}7.38(8) \\
(9,10)\end{array}$ \\
\hline 0.60 & $\begin{array}{l}6.79(10) \\
(10,14)\end{array}$ & $\begin{array}{l}6.97(12) \\
(14,15)\end{array}$ & $\begin{array}{l}6.58(10) \\
(10,14)\end{array}$ & $\begin{array}{l}7.62(12) \\
(14,15)\end{array}$ & $\begin{array}{l}6.88(10) \\
(10,14)\end{array}$ & $\begin{array}{l}7.41(12) \\
(14,15)\end{array}$ \\
\hline 0.61 & $\begin{array}{l}6.92(9) \\
(10,12)\end{array}$ & $\begin{array}{l}6.96(11) \\
(13,14)\end{array}$ & $\begin{array}{l}7.10(9) \\
(10,12)\end{array}$ & $\begin{array}{l}7.49(11) \\
(13,14)\end{array}$ & $\begin{array}{l}7.34(9) \\
(10,12)\end{array}$ & $\begin{array}{l}7.42(11) \\
(13,14)\end{array}$ \\
\hline 0.62 & $\begin{array}{l}6.87(13) \\
(14,18)\end{array}$ & $\begin{array}{l}6.95(13) \\
(15,17)\end{array}$ & $\begin{array}{l}6.71(13) \\
(14,18)\end{array}$ & $\begin{array}{l}7.28(13) \\
(15,17)\end{array}$ & $\begin{array}{l}6.99(13) \\
(14,18)\end{array}$ & $\begin{array}{l}7.40(13) \\
(15,17)\end{array}$ \\
\hline 0.63 & $\begin{array}{l}6.95(12) \\
(14,16)\end{array}$ & $\begin{array}{l}7.00(13) \\
(16,17)\end{array}$ & $\begin{array}{l}7.10(12) \\
(14,16)\end{array}$ & $\begin{array}{l}7.33(10) \\
(12,13)\end{array}$ & $\begin{array}{l}7.34(12) \\
(14.16)\end{array}$ & $\begin{array}{l}7.49(13) \\
(16,17)\end{array}$ \\
\hline 0.64 & $\begin{array}{l}5.88(11) \\
(10,18)\end{array}$ & $\begin{array}{l}7.01(9) \\
(11,12)\end{array}$ & $\begin{array}{l}5.41(7) \\
(8,10)\end{array}$ & $\begin{array}{l}7.10(9) \\
(11,12)\end{array}$ & $\begin{array}{l}5.53(7) \\
(8,10)\end{array}$ & $\begin{array}{l}7.34(9) \\
(11,12)\end{array}$ \\
\hline 0.65 & $\begin{array}{l}6.58(13) \\
(14,20)\end{array}$ & $\begin{array}{l}7.04(12) \\
(15,16)\end{array}$ & $\begin{array}{l}5.69(13) \\
(16,18)\end{array}$ & $\begin{array}{l}7.10(12) \\
(15,16)\end{array}$ & $\begin{array}{l}5.76(13) \\
(16,18)\end{array}$ & $\begin{array}{l}7.34(12) \\
(15,16)\end{array}$ \\
\hline
\end{tabular}

Optimum code $\left\{\begin{array}{l}h_{+1}, h_{+2} \\ h_{-1}, h_{-2}\end{array}\right\}=\left\{\begin{array}{l}I_{+1} / q, I_{+2} / q \\ I_{-1} / q, I_{-2} / q\end{array}\right\}$

and $A_{1}=I_{+1}+I_{-1}, A_{2}=I_{+2}+I_{-2}$

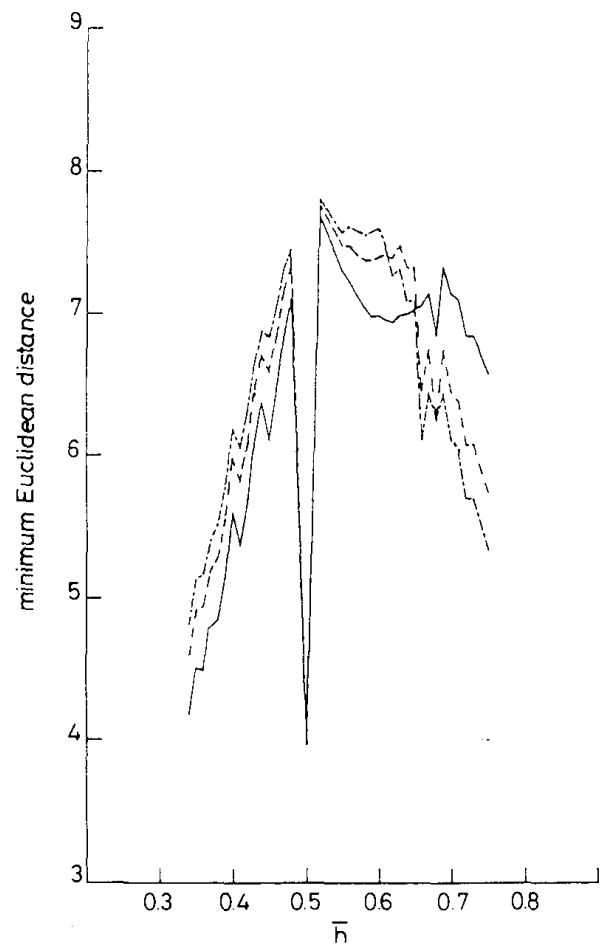

Fig. 1 Minimum Euclidean distances against averaged modulation index $\bar{h}$ for asymmetric $M H P M$ with $K=2$

- LP RC 


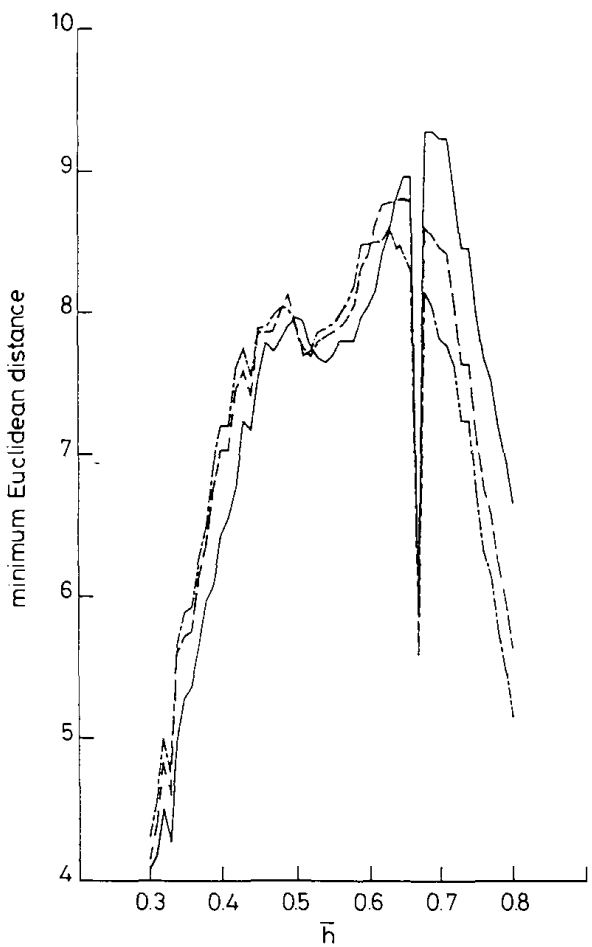

Fig. 2 Minimum Euclidean distances against averaged modulation index $\bar{h}$ for asymmetric $M H P M$ with $K=3$

$\begin{array}{ll}- & \text { LP } \\ -- & \text { RC }\end{array}$

$\ldots$ HCS

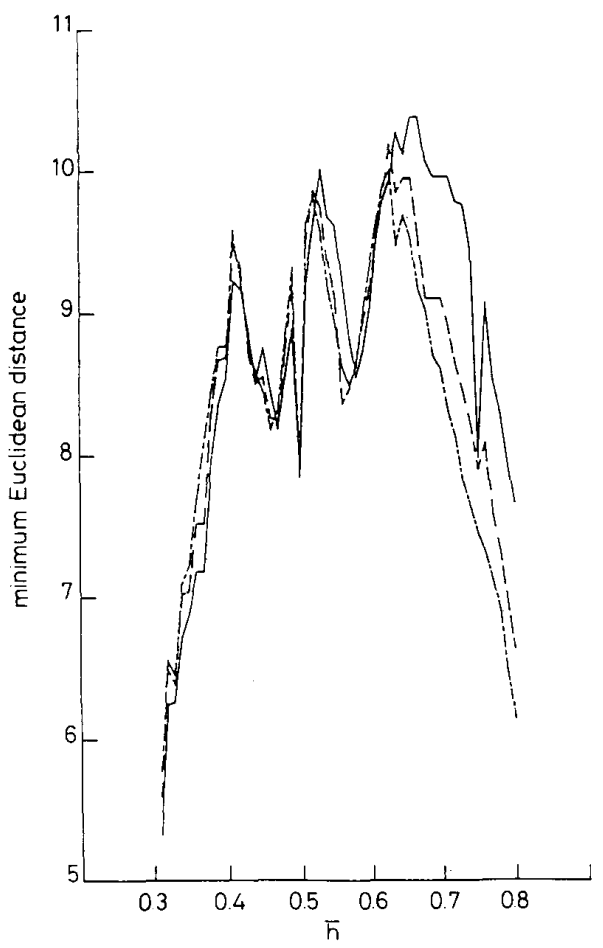

Fig. 3 Minimum Euclidean distances against averaged modulation index $\bar{h}$ for asymmetric $M H P M$ with $K=4$

\begin{tabular}{ll}
- & LP \\
\hdashline- & RC \\
$\ldots-$ & HCS
\end{tabular} tion are larger than those for LP and HCS functions when $\bar{h}$ is smaller than 0.41 . The peak of 9.59 for the minimum Euclidean distance of RC phase MHPM scheme is the best choice when $\bar{h}$ is required to be less than 0.5. On the other hand, LP phase pulse function will be the best choice when $\bar{h}$ is allowed to exceed 0.64 . Also, it is clear that the minimum Euclidean distances for LP have two peaks of 10.01 and 10.39 for $\bar{h}=0.53$ and $\bar{h}=$ 0.66 , respectively. However, because the coding gain difference is only $0.16 \mathrm{~dB}$ between these two peaks, but the bandwidth for $\bar{h}=0.53$ is apparently much smaller, thus the LP function with modulation index set with $\bar{h}=0.53$ seems to be a very attractive choice when considering both the bandwidth and power efficiencies.

By comparing Figs. 1-3, it is quite clear that the RC phase pulse function is the best choice when $\bar{h}$ is required to be less than 0.5. Also, the HCS phase pulse function is less power efficient than LP or RC phase pulse functions in general. Furthermore, a comparison of Figs. 2 and 3 indicates that the distances of $4-h$ schemes with $\bar{h}=0.53$ can be larger than those of $3-h$ schemes with $\bar{h}=0.68$. This means with $4-h$ schemes better error probability performance can be achieved than with the best of 3- $h$ schemes, even with significantly less bandwidth but at the price of increased complexity because complexity increases with $K$. It is thus quite clear that there exist many asymmetric MHPM schemes which can be chosen in system design when the trade-offs among bandwidth and power efficiencies and system complexity are considered.

\section{Performance analysis for partial response cases}

We now analyse the performance for partial response MHPM with asymmetric indices and linear phase pulse function as described in eqn. 6. The minimum Euclidean distances for the best combinations of modulation indices with $K=2,3$ and 4 have been calculated for linear phase partial response multi- $h$ schemes. The results for full response MHPM systems are listed in columns 1 and 2 of Tables 5-7, while those for partial response MHPM

Table 5: Minimum Euclidean distances for 2-h linear phase MHPM systems

\begin{tabular}{|c|c|c|c|c|}
\hline \multirow[t]{3}{*}{$q$} & \multicolumn{4}{|c|}{ Minimum Euclidean distance $\left(A_{1}, A_{2}\right)$} \\
\hline & \multicolumn{2}{|c|}{ full response } & \multicolumn{2}{|c|}{ partial response } \\
\hline & conv. & asym. & conv. & asym. \\
\hline 4 & 5.58 & 7.11 & 4.36 & 8.41 \\
\hline & $(4,6)$ & $(4,5)$ & $(4,6)$ & $(6,7)$ \\
\hline 5 & 6.14 & 7.25 & 6.85 & 8.83 \\
\hline 6 & 6.90 & 7.36 & 7.39 & 8.69 \\
\hline & $(6,8)$ & $(6,7)$ & $(8,10)$ & $(9,11)$ \\
\hline 7 & 6.65 & 7.45 & 7.90 & 8.60 \\
\hline & $(6,10)$ & $(7,8)$ & $(10,12)$ & $(11,13)$ \\
\hline 8 & 7.10 & 7.51 & 8.41 & 9.03 \\
\hline & $(8,10)$ & $(8,9)$ & $(12,14)$ & $(13,14)$ \\
\hline 9 & 6.92 & 7.57 & 8.85 & 8.92 \\
\hline & $(10,12)$ & $(9,10)$ & $(14,16)$ & $(15,16)$ \\
\hline 10 & 7.25 & 7.61 & 8.83 & 8.83 \\
\hline & $(10,12)$ & $(10,11)$ & $(16,18)$ & $(16,18)$ \\
\hline 11 & 7.14 & 7.64 & 8.76 & 9.02 \\
\hline & $(10,14)$ & $(11,12)$ & $(18,20)$ & $(18,19)$ \\
\hline 12 & 7.36 & 7.67 & 8.69 & 9.03 \\
\hline & $(12,14)$ & $(12,13)$ & $(18,20)$ & $(20,21)$ \\
\hline 13 & 7.28 & 7.70 & 8.64 & 8.95 \\
\hline & $(12,16)$ & $(13,14)$ & $(20,24)$ & $(22,23)$ \\
\hline
\end{tabular}

IEE PROCEEDINGS-I, Vol. 139, No. S, OCTOBER 1992 
Table 6: Minimum Euclidean distances for 3-h linear phase MHPM systems

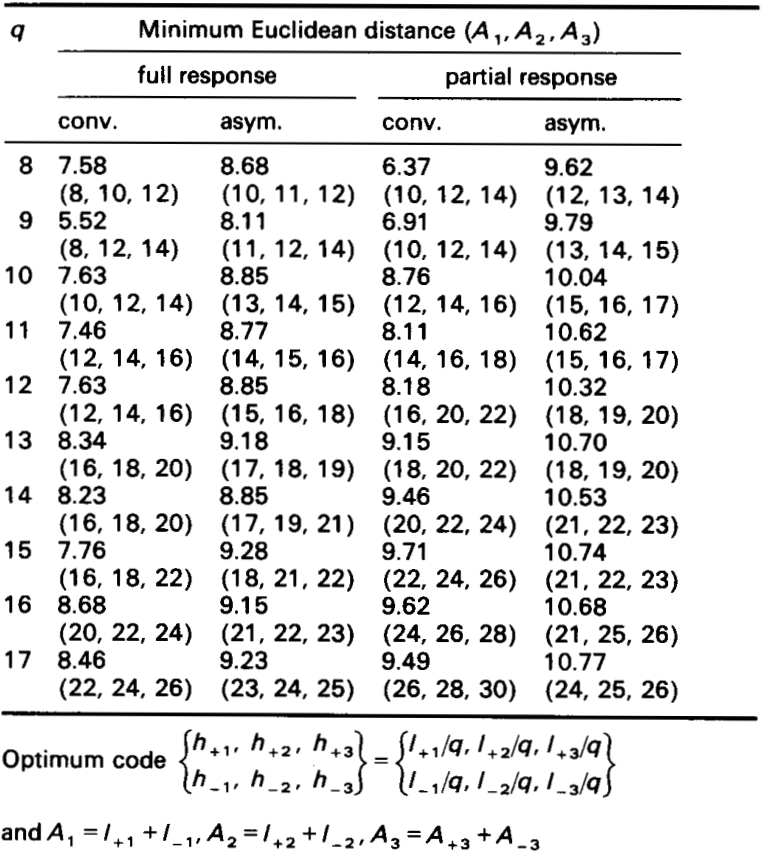

systems are listed in columns 3 and 4. From these tables, it is clear that the minimum Euclidean distances for asymmetric MHPM schemes are always larger than those of conventional symmetric MHPM, for all values of $q$ for which the calculation has been performed. It is also revealed that the minimum Euclidean distances are much less dependent on the choice of $q$ when asymmetric modulation indices are used. Furthermore, it is interesting to find that most of the best combinations of $h$ used for partial/full response asymmetric MHPM systems are different. From Tables 6 and 7, we find that the distances of asymmetric MHPM with $K=3$ are very close to those of conventional symmetric MHPM with $K=4$. Since the implementation complexity of a multi- $h$ system increases with $K$, this means asymmetric MHPM can be used to obtain even better error probability performance, compared to conventional symmetric MHPM but with less complexity. For example, for an asymmetric 3 - $h$ scheme with $q=17$, we can obtain a minimum Euclidean distance very close to that of the best conventional 4- $h$ scheme with $q=20$.

In Fig. 4, the minimum Euclidean distances against $q$ are plotted with $K=2,3$ and 4 for both linear phase full response and partial response MHPM's with asymmetric modulation indices. It is easy to see in this Figure that,

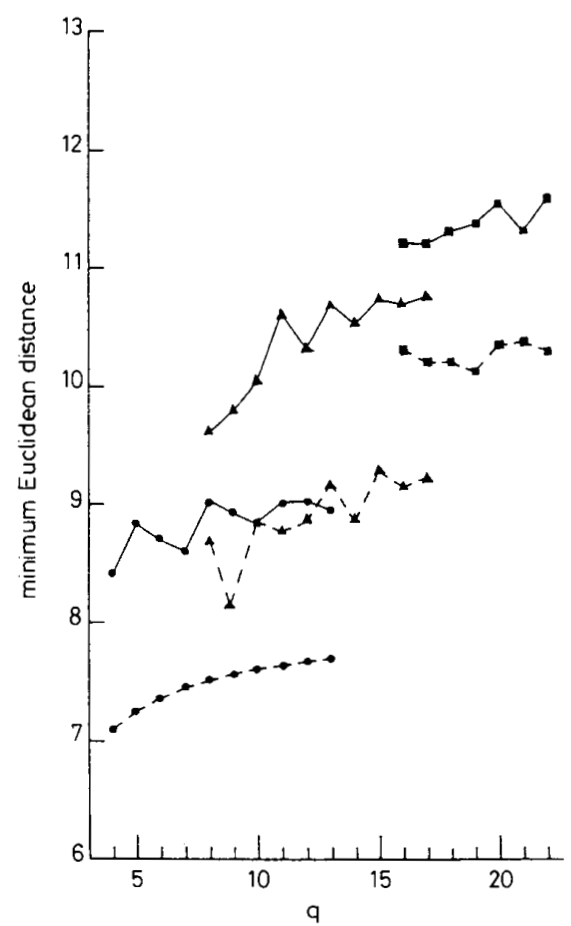

Fig. 4 Comparison of minimum Euclidean distances for full response and partial response $M H P M$ with asymmetric modulation indices

Partial response

...... Full response

$K=2 \Delta K=3 \square K=4$

Table 7: Minimum Euclidean distances for 4-h linear phase MHPM systems

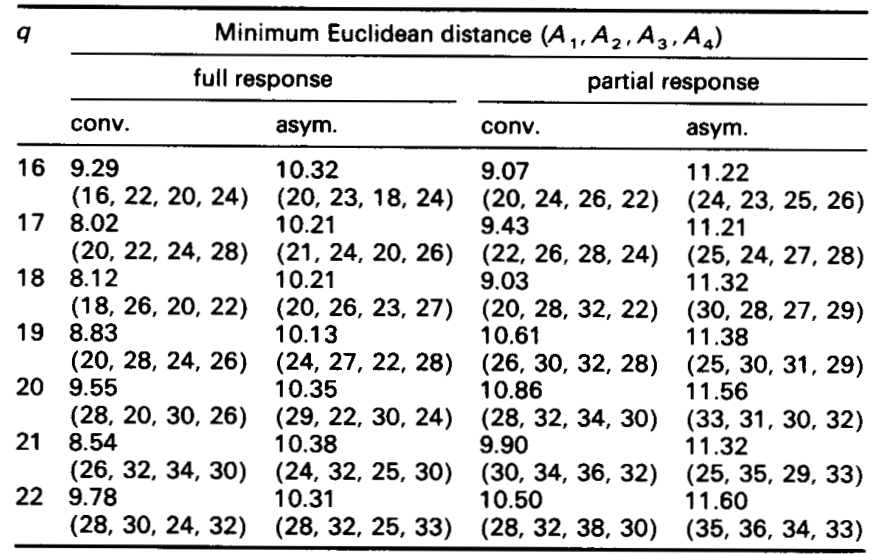

$$
\begin{aligned}
& \text { Optimum code }\left\{\begin{array}{ll}
h_{+1}, h_{+2}, & h_{+3}, h_{+4} \\
h_{-1}, & h_{-2}, h_{-3}, h_{-4}
\end{array}\right\}=\left\{\begin{array}{ll}
I_{+} / q, I_{+2} / q, I_{+3} / q, I_{+4} / q \\
I_{-1} / q, I_{-2} / q, I_{-3} / q, I_{-4} / q
\end{array}\right\} \\
& \text { and } A_{7}=I_{+1}+I_{-1}, A_{2}=I_{+2}+I_{-2}, A_{3}=I_{+3}+I_{-3}, A_{4}=I_{+4}+I_{-4}
\end{aligned}
$$


for asymmetric MHPM schemes, partial response provides a better performance compared to full response for $K$ varing from 2 to 4 . Furthermore, it can be found that the Euclidean distances of linear phase partial response asymmetric MHPM with $K=2$ and $K=3$ are very close to those of full response asymmetric MHPM with $K=3$ and $K=4$, respectively.

\section{Conclusion}

In this paper the new concept of asymmetric indices recently proposed for MHPM is extended to partial response systems and different phase pulse functions. It is found that significant improvements in power efficiency are possible, and a full range of design trade-offs among bandwidth and power efficiencies and complexity are available for system optimisation. For full response MHPM systems, the RC phase pulse function turns out to be a very good choice when the bandwidth-power trade-off is considered for average indices less than 0.5 . With asymmetric modulation indices, partial response is shown to give better performance than full response at the price of increased system complexity.

\section{References}

1 AULIN, T., and SUNDBERG, C.E.: 'Continuous phase modulation - Part I: Full response signaling', IEEE Trans., 1981, COM-29, pp. 196-209
2 AULIN, T., RYDBECK, N., and SUNDBERG, C.E.: 'Continuous phase modulation - Part II: Partial response signaling', IEEE Trans., 1981, COM-29, pp. 210-225

3 UNGERBOECK, G.: "Channel coding with multilevel/phase signals', IEEE Trans., 1982, IT-28, pp. 55-67

4 ANDERSON, J.B., and TAYLOR, D.P.: 'A bandwidth-efficient class of signal-space codes', IEEE Trans., 1978, IT-24, pp. 703-712

5 ANDERSON, J.B., and DE BUDA, R.: 'Better phase-modulation error performance using trellis phase codes', Electron. Lett., 1976, 12, pp. 587-588

6 ANDERSON, J.B., and TAYLOR, D.P.: 'Trellis phase modulation coding: minimum distance and spectral results'. Proc. Electron. Aerospace Syst. Conf., Arlington, VA, Sept. 25-28, 1977, pp. 29-1A29-1G

7 ANDERSON, J.B., TAYLOR, D.P., and LEREIM, A.T.: 'A class of trellis phase modulation codes for coding without bandwidth expansion'. Proc. Int. Conf. on Communications, Toronto, Canada, June 4-7, 1978, pp. 50.3.1-50.3.5

8 ANDERSON, J.B., SUNDBERG, C.E, AULIN, T., and RYDBECK, N.: 'Power bandwidth performance of smoothed phase modulation codes', IEEE Trans., 1981, COM-29, pp. 187-195

9 ANDERSON, J.B.: 'Simulated error performance of multi-h phase codes', IEEE Trans., 1981, IT-27, pp. 357-362

10 AULIN, T., and SUNDBERG, C.E.: 'Minimum Euclidean distance and power spectrum for a class of smoothed phase modulation codes with constant envelope', IEEE Trans., 1982, COM-30, pp. 1721-1729

11 HWANG, H.K., LEE, L.S., and CHEN, S.H.: 'Multi-h phase-coded modulations with asymmetric modulation indices', IEEE J. Selected Areas Commun., 1989, SAC-7, pp. 1450-1461

12 LIND, L.F., and REDWOOD-SAWYERR, J.A.S.: 'Error performance of multi-h partial-response phase codes', IEE Proc. F, Commun., Radar \& Signal Processing, 1987, 134, pp. 405-415

13 WILSON, S.G., and GAUS, R.C.: 'Power spectra of multi- $h$ phase codes', IEEE Trans., 1981, COM-29, pp. 250-256 